\title{
ERRATUM
}

\section{Room-temperature generation of giant pure spin currents using epitaxial $\mathrm{CO}_{2} \mathrm{FeSi}$ spin injectors}

Takashi Kimura, Naoki Hashimoto, Shinya Yamada, Masanobu Miyao and Kohei Hamaya

NPG Asia Materials (2012) 4, e13; doi:10.1038/am.2012.24; published online 30 March 2012

Correction to: NPG Asia Materials 4, e9; doi:10.1038/am.2012.16; published online 9 March 2012

The volume number for the original PDF version of this article was incorrect. The correct volume number is 4 . 